\title{
Right ventricular outflow tract resection for treatment of refractory ventricular tachycardia in a 2-year-old child
}

\author{
S. Kanaan, MD, K. Shamsuddin, MD, M. Silka, MD, and W. Wells, MD, Los Angeles, Calif
}

$\mathrm{V}$ entricular tachycardia (VT) of right ventricular origin in young children might be due to Purkinje cell tumors, arrhythmogenic right ventricular dysplasia (ARVD), or Uhl's anomaly, or it might be idiopathic. ${ }^{1}$ Surgical intervention has been used in adults when medical or catheter ablative therapy is ineffective. We describe a unique cause of VT of right ventricular outflow tract (RVOT) origin in a young child that was successfully managed with surgical resection.

\footnotetext{
From the Department of Cardiothoracic Surgery, Children's Hospital of Los Angeles, Keck School of Medicine, University of Southern California, Los Angeles, Calif.

Address for reprints: S. Kanaan, MD, Department of Cardiothoracic Surgery, University of Southern California, 1520 San Pablo St, HCC2 Suite 4300, Los Angeles, CA 90033 (E-mail: skanaan@earthlink.net).

J Thorac Cardiovasc Surg 2005;130:948-9

$0022-5223 / \$ 30.00$

Copyright $(\odot 2005$ by The American Association for Thoracic Surgery

doi:10.1016/j.jtcvs.2005.03.031
}

\begin{abstract}
Clinical Summary
A 2-year-old child presented with syncope and near-incessant VT at a rate of 270 beats $/ \mathrm{min}$. The tachycardia was notable for subtle irregularities in both cycle length and QRS morphology (Figure 1). However, all QRS complexes suggested an RVOT origin, with positive vectors in leads II, III, and aVF. Transthoracic echocardiography showed marked dilation of the RVOT, which was confirmed by means of cardiac magnetic resonance imaging. At electrophysiologic testing, the arrhythmia could not be terminated with overdrive pacing but did temporarily stop with adenosine. Activation mapping defined the onset of activation at the anterior RVOT immediately inferior to the pulmonary valve annulus. Catheter probing of this area resulted in marked acceleration of the arrhythmia and hypotension. Because of the marked dilation and attenuation of tissue in this region and hemodynamic instability with mapping, catheter ablation was not performed.

Despite pharmacologic therapy with amiodarone, lidocaine, and class $1 \mathrm{C}$ agents, recurrences of sustained VT with hemodynamic compromise persisted. Thus surgical intervention was recommended, with anticipation that discrete myocardial tumors would be identified.

At the time of the operation, the anterior free wall of the RVOT was dilated and visibly thin, and it collapsed when the patient was
\end{abstract}

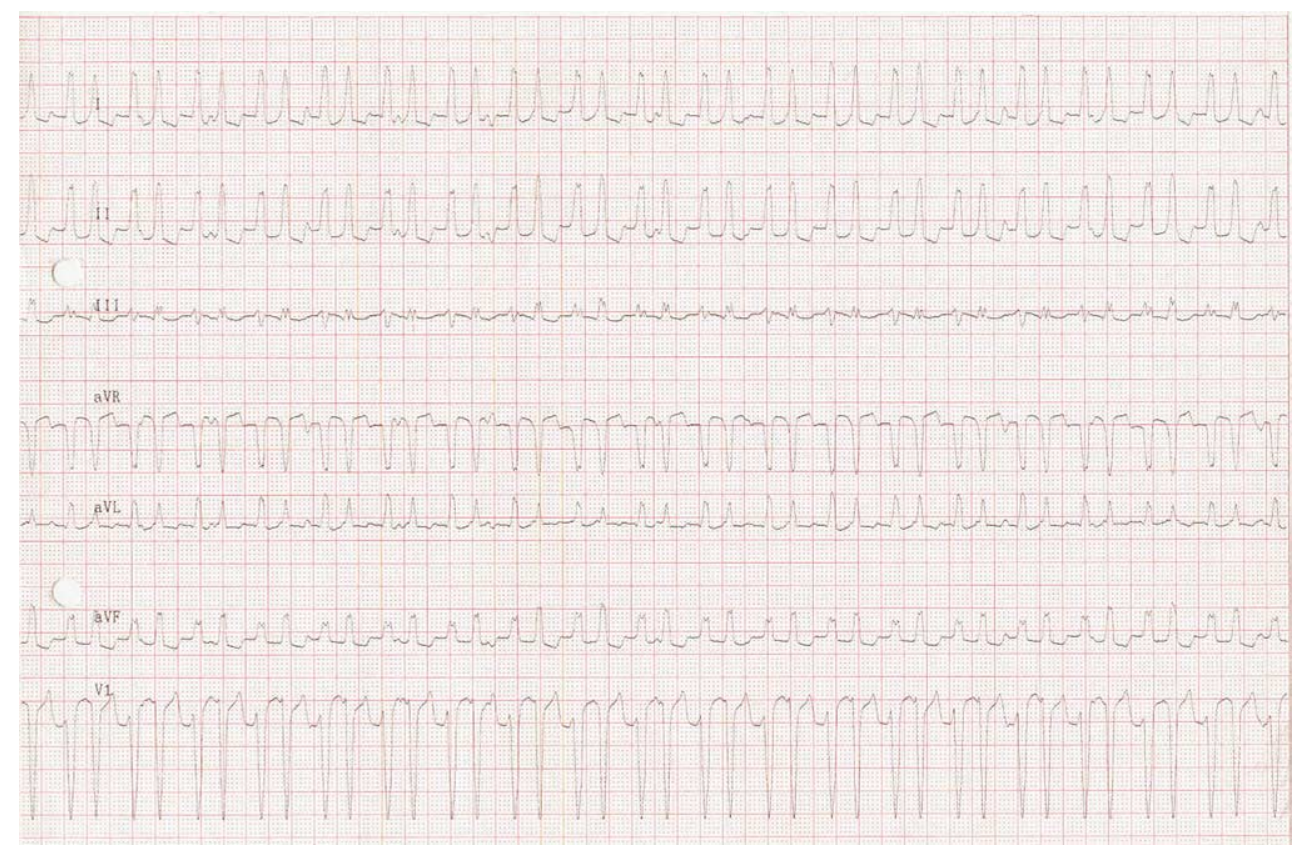

Figure 1. Electrocardiographic leads $\mathrm{I}$, aVF, and V1 recorded at $100 \mathrm{~mm} / \mathrm{s}$ during electrophysiology testing. The high right atrial electrocardiogram confirms the diagnosis of VT with ORS and atrial dissociation. This tracing also emphasizes the irregular cycle length of the tachycardia and variations in ORS morphology. 


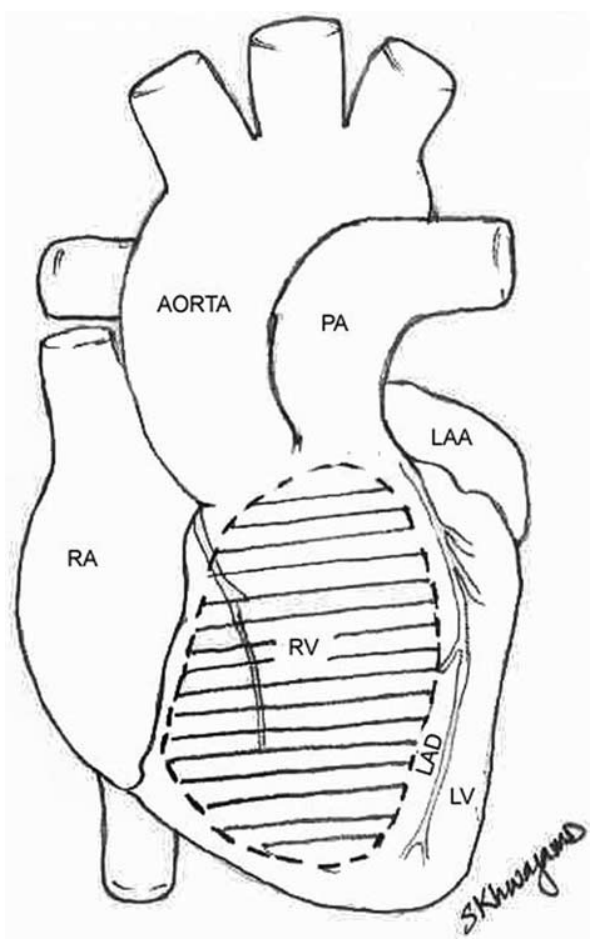

Figure 2. Portion of the anterior wall of the right ventricle resected at operation. $R A$, Right atrium; $P A$, pulmonary artery; $L A A$, left atrial appendage; $R V$, right ventricle; $L V$, left ventricle; $L A D$, left anterior descending artery.

started on bypass. A wide resection of the attenuated tissue was performed (Figure 2), and the anterior free wall of the right ventricle was reconstructed with glutaraldehyde-treated autologous pericardium. The postoperative course was unremarkable, and the patient was discharged 3 days later. Amiodarone $(50 \mathrm{mg} / \mathrm{d})$ was discontinued 2 weeks after the operation. After several months of follow-up, the patient remains free of arrhythmias and all medications.

Pathologic sections from the RVOT demonstrated disorganized and abnormally oriented myocytes with enlarged nuclei. There were no fibrofatty replacement of myocardium, no inflammatory cells, and no ectopic Purkinje cells.

\section{Discussion}

This case represents a unique pathologic substrate as a cause of VT in a young child. Given the cellular pleomorphism and disorganization, the irregular cycle length of VT, and the response to adenosine, a multifocal automatic or triggered origin of the arrhythmia from the RVOT outflow tract is probable. By pathologic study, most of the defined causes of tachycardia were excluded: no ectopic Purkinje or inflammatory cells were identified, and there was no replacement of the myocardium with fibrous or fatty tissue, as would be found in Uhl's anomaly or ARVD. Additionally, the very rapid and incessant nature of the tachycardia would be inconsistent with characteristics of idiopathic right VT.

ARVD is an inherited progressive cardiomyopathy caused by replacement of the myocardium with fibrofatty tissue. ${ }^{1,2}$ It has a highly variable presentation, including right heart failure, right VT, or sudden death. ${ }^{3,4}$ Its prevalence is estimated to be 1 in 5000, with familial cases accounting for $30 \%$ to $50 \%$. $^{3}$ Surgical intervention has been described as a curative treatment for VT associated with ARVD in adults. ${ }^{4,5}$ Misaki and colleagues ${ }^{5}$ performed resection of the right ventricle and cryoablation in 8 male adults (average age, 36 years), with no surgical or postoperative deaths. Only a few case reports exist of ARVD in children.

RVOT tachycardia is characterized as a benign electrical disease with no structural abnormality. ${ }^{1}$ Like ARVD, RVOT tachycardia can occur in children, but no reports exist of surgical resection for treatment. Whether the 2 conditions are separate entities or part of a disease spectrum is not clear, but it is possible that RVOT tachycardia represents an early form of ARVD.

We describe an unusual case of a 2-year-old child with polymorphic VT originating from the RVOT. In spite of dilation of the RVOT and attenuation of the regional myocardium, pathologic changes pathognomonic for the known forms of VT in young children were not present. We conjecture that with time, this child might experience further pathologic changes more consistent with those seen in ARVD and that this case represents an early manifestation of an evolving pathologic process.

\section{References}

1. O'Donnell D, Cox D, Bourke J, Mitchell L, Furniss S. Clinical and electrophysiological differences between patients with arrhythmogenic right ventricular dysplasia and right ventricular outflow tract tachycardia. Eur Heart J. 2003;24:801-10.

2. Marcus FI, Fontaine GH, Guiraudon G, Frank R, Laurenceau JL, Malergue S, et al. Right ventricular dysplasia. Circulation. 1982;65: 384-98.

3. Tabib A, Loire R, Chalabreysse L, Meyronnet D, Miras A, Malicier D, et al. Circumstances of death and gross and microscopic observations in a series of 200 cases of sudden death associated with arrhythmogenic right ventricular cardiomyopathy and/or dysplasia. Circulation. 2003; 108:3000-5.

4. Motta P, Mossad E, Savage R. Right ventricular exclusion surgery for arrhythmogenic right ventricular dysplasia and cardiomyopathy. Anesth Analg. 2003;96:1598-602.

5. Misaki T, Watanabe G, Iwa T, Tsubota M, Ohtake H, Yamamoto K, et al. Surgical treatment of arrhythmogenic right ventricular dysplasia: long-term outcome. Ann Thorac Surg. 1994;58:1380-5. 